EPJ Web of Conferences 22, 00002 (2012)

DOI: $10.1051 /$ epjconf/20122200002

(C) Owned by the authors, published by EDP Sciences, 2012

\title{
Symmetry and group theory throughout physics
}

\author{
J. Villain \\ Theory Group, ESRF, 6 rue Jules Horowitz, 38000 Grenoble, France
}

\begin{abstract}
As noticed in 1884 by Pierre Curie [1], physical properties of matter are tightly related to the kind of symmetry of the medium. Group theory is a systematic tool, though not always easy to handle, to exploit symmetry properties, for instance to find the eigenvectors and eigenvalues of an operator. Certain properties (optical activity, piezoelectricity) are forbidden in molecules or crystals of high symmetry. A few theorems (Noether, Goldstone) establish general relations between physical properties and symmetry. Applications of group theory to condensed matter physics, elementary particle physics, quantum mechanics, electromagnetism are reviewed. Group theory is not only a tool, but also a beautiful construction which casts insight into natural phenomena.
\end{abstract}

\section{SYMMETRY ET PHYSICAL PROPERTIES}

Life is possible on the Earth because of the physical properties of the molecules around us: $\mathrm{O}_{2}, \mathrm{~N}_{2}$, $\mathrm{H}_{2} \mathrm{O}$... These physical properties depend much on symmetry. Thus, $\mathrm{O}_{2}$ and $\mathrm{N}_{2}$ molecules are symmetric with respect to their center (Fig. 1). This implies that their electric dipole moment is zero. This property is not altered when the molecule vibrates. Therefore, pure, dry air does not absorb the infrared radiation emitted by the Earth. ${ }^{1}$ Therefore the greenhouse effect results only from water and other less symmetric molecules. Oxygen and nitrogen do not contribute to the greenhouse effect. This is an essential result, although its proof is elementary.

Another example concerns a material which is currently highly fashionable, graphene [2]. Its electronic structure has a remarkable property, namely the conduction band and the valence band are separated, except for a discrete set of points of the reciprocal space, where they touch. This is in contrast with the situation in a metal or an insulator or semiconductor. In Appendix A this is shown to result from symmetry.

These are examples of the considerable simplification brought by symmetry in physical problems. In many cases, symmetry is more difficult to exploit, but makes the analysis easier. Then the concept of group and group theory may be useful.

In the following introduction, we shall try to find the right compromise between too superficial and too technical considerations.

\section{DEFINITION OF GROUPS}

A group is a set of elements $g_{i}$ with a multiplication rule which associates to any two elements $g_{i}$, $g_{j}$ the product $g_{i} \cdot g_{j}$ or $g_{i} g_{j}$. There is a neutral element or 'unity' 1 such that $g_{i} .1=1 . g_{i}=g_{i}$. Each element $g_{i}$ should have an inverse $g_{i}^{-1}$ such that $g_{i} \cdot g_{i}^{-1}=g_{i}^{-1} \cdot g_{i}=1$. Multiplication is associative, $g_{i} \cdot\left(g_{j} \cdot g_{k}.\right)=\left(g_{i} \cdot g_{j}.\right) \cdot g_{k}$. It may or may not be commutative. A group with a commutative multiplication

\footnotetext{
${ }^{1}$ This argument ignores the electrons, which can be responsible for an electric dipole moment. However, the corresponding emission and absorption lines have a higher frequency. They can absorb the (visible) light coming from the sun, but not the infrared light reemitted by the Earth.

This is an Open Access article distributed under the terms of the Creative Commons Attribution-Noncommercial License 3.0, which permits unrestricted use, distribution, and reproduction in any noncommercial medium, provided the original work is properly cited.
} 

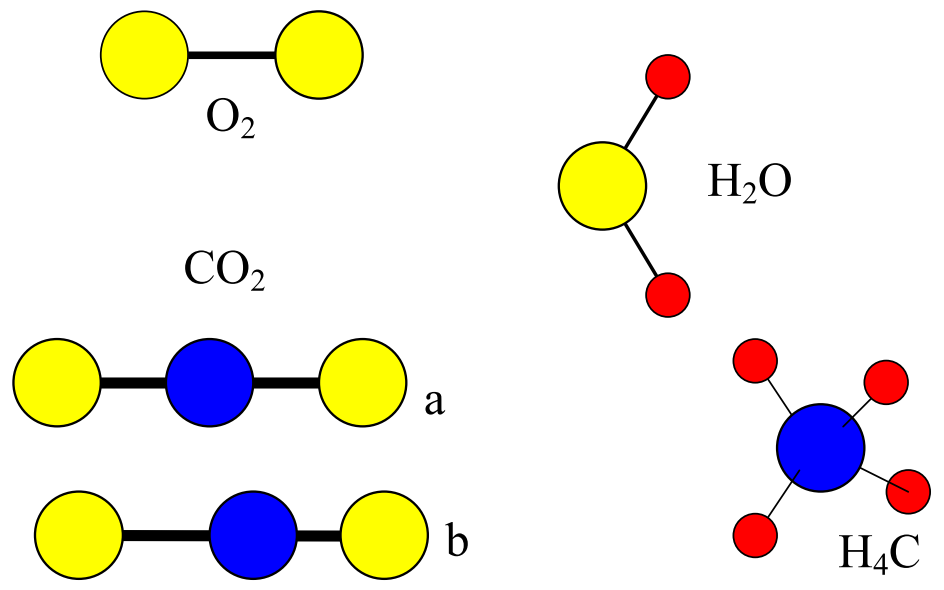

Figure 1. symmetry of various molecules. Oxygen and nitrogen are centrosymmetric and remain such when they vibrate. Therefore, they can neither absorb nor emit infrared radiation. Water, carbon dioxyde and methane have a variable electric dipole moment when they vibrate, and thus can absorb and emit infrared radiation. Carbon dioxyde has a vanishing average electric dipole moment (a) but acquires a dipole moment when it vibrates (b). Methane a has an analogous property.

$\left(g_{i} \cdot g_{j}=g_{j} \cdot g_{i}\right)$ is called abelian. A group may be finite or infinite. A more rigorous treatment can be found in the chapter by Canals and Schober.

The transformations which conserve a system (e.g. a polyhedron, a crystal...) form a group. The unity is the 'identical transformation' 1 , which just transforms any object into itself. For example, if $I$ is the symmetry with respect to a point $\mathrm{O}$, it forms with the identical transformation a group of 2 elements. This group has been implicitly considered in the previous section but for such a simple problem the concept of group and group theory are not necessary.

The group of the transformations which leave an object unchanged is called the symmetry of this object. This word 'symmetry' has thus a broader meaning than in usual life.

\section{A LIST OF GROUPS USED BY PHYSICISTS}

The following list may be useful to the reader as a reminder.

1) The group of rotations around a given point in the three-dimensional space is called $\mathrm{SO} 3$ or $\mathrm{SO}(3)$. For instance, in the non-relativistic treatment of the hydrogen atom, the Hamiltonian is invariant under SO3 and this facilitates the search for the wave functions, as will be seen. More generally it is possible to define the group $\mathrm{SO}(n)$.

2) The group $\mathrm{O} 3$ or $\mathrm{O}(3)$ is that of proper and improper rotations in the three-dimensional space. It can be generated from SO3 by adding the centrosymmetry. An improper rotation is the product of a rotation by the symmetry with respect to a point.

3) The group of three dimensional translations is useful to describe the propagation of a free, nonrelativistic particle.

4) The group of rotations et translations in three dimensions.

5) The group of the translations which conserve a crystal is a sub-group of (3).

6) The group of the transformations which conserve a crystal is called the space group of the crystal. It is a sub-group of (4). Each element of a space group is the product of a translation by a rotation $\mathcal{R}$ around an axis that passes through a given point $\mathrm{O}$.

7) The point group of a crystal is the group of the rotations $\mathcal{R}$ defined above. Point groups are finite groups. 


\section{Contribution of Symmetries in Condensed Matter}

8) Lorentz group, Poincaré group. They are generated by 'Lorentz transformations' used in relativistic physics, where time $t$ and space coordinates $x, y, z$ cannot be transformed independently. For instance, a motion along the $x$ axis is described by the Lorentz transformation

$$
x^{\prime}=(x-v t) /\left(1-\beta^{2}\right)^{1 / 2} ; t^{\prime}=(t-v x / c) /\left(1-\beta^{2}\right)^{1 / 2} ; y^{\prime}=y ; z^{\prime}=z
$$

where $\beta=v / c$. This transformation conserves the quantity

$$
d s^{2}=d x^{2}+d y^{2}+d z^{2}-c^{2} d t^{2}
$$

while a three-dimensional rotation or translation conserves

$$
d s^{2}=d x^{2}+d y^{2}+d z^{2}
$$

Any transformation which conserves (3.2) is a Lorentz transformation. The Lorentz group and the Poincaré group correspond to different selections of Lorentz transformations as will be seen.

9) $\mathrm{SU} 2, \mathrm{SU} 3 \ldots \mathrm{SU} n$ or $\mathrm{SU}(n) .=$ group of $n \times n$ unitary matrices of determinant 1.

$10) \mathrm{U}(n)$ is the group of unitary $n \times n$ matrices. For instance, $\mathrm{U}(1)$ is formed by the numbers $\exp (i \varphi)$.

Continuous groups as $\mathrm{O}(n)$ are parts of a more general class called Lie groups.

The concept of group is present in many domains of physics. What is it good for? The following examples give an idea of the answer.

\section{CRYSTALLOGRAPHY}

A crystal is the thermodynamically stable form of most materials at low temperature. This makes them particularly important. The symmetry of crystals is described in detail in the chapters by Grenier and Ballou, Perez-Mato et al. and Tasci et al.

The symmetry of a crystal is characterized by its space group, defined in the previous section. Certain properties depend only on the point group. On the other hand, a crystal with a single atom per unit cell (like iron and many other elements) is called a Bravais lattice. There are 32 point groups and 230 space groups, as independently shown by Schönflies and by Fedorov in 1890. There are 14 Bravais lattices, as shown by Bravais in 1848. These numbers result from the solution of a mathematical problem, comparable to the descriptive enumeration of the 5 regular polyhedra by Euclid ${ }^{2}$.

What are point groups and space groups good for? Certain physical properties do or do not exist according to the crystal symmetry. An example is pyroelectricity, characterized by a non vanishing electric dipole moment $\mathbf{P}$ in each unit cell. In a pyroelectric crystal, any symmetry operation must preserve the direction of polarisation. The only possible rotation axis is therefore parallel to $\mathbf{P}$. Moreover, a mirror plane perpendicular to $\mathbf{P}$ is forbidden. This is only possible for point groups $C_{n}, C_{n v}$, and $C_{1 h}$. The group $C_{1}$ is constituted by the unity alone, and is triclinic. The group $C_{n}$ implies a rotation axis of order $n(n=2,3,4,5)$ and nothing else. The group $C_{n v}$ in addition contains a 'mirror' (or symmetry plane) parallel to this axis. The group $C_{1 h}$ is made of unity and a mirror.

Another example is birefringence. It is present in all non-cubic crystals. It is related to electric permittivity tensor $\epsilon$ defined by $\mathbf{D}=\epsilon \mathbf{E}$, where $\mathbf{E}$ and $\mathbf{D}$ are respectively the electric field and displacement. A crystal is birefringent if the 3 eigenvalues of the matrix $\epsilon$ are not all equal.

Matrices? They are usual in physics, and the physicist likes to know their eigenvalues and eigenvectors. Group theory can help.

\footnotetext{
2 To give an idea of the proof, it will be shown that any rotation of angle $\varphi<\pi / 3$ is forbidden. If a Bravais lattice were invariant under such a rotation, this lattice would contain 3 points $\mathrm{ABC}$ such that $\mathrm{AB}=\mathrm{BC}$ with the angle $\mathrm{ABC}$ equal to $\varphi<\pi / 3$. The length $\mathrm{AC}$ would thus be smaller than $\mathrm{AB}$ and $\mathrm{BC}$. But the lattice would then also contain a point $\mathrm{D}$ with $\mathrm{AD}=\mathrm{AC}$, the angle $\mathrm{DAC}$ being smaller than $\pi / 3$. The length $\mathrm{DC}$ would be smaller than $\mathrm{AD}$ and $\mathrm{AC}$. By recursion, one can thus find lengths as small as one wants, and this is not acceptable.
} 


\section{EIGENVECTORS OF AN OPERATOR AND IRREDUCIBLE REPRESENTATIONS OF A GROUP}

Group theory facilitates research and classification of the eigenvectors (and therefore of the eigenvalues) of a matrix or an operator. This operator can be a Hamiltonian for instance.

A well known case, where group theory is underlying, is that of a $N \times N$ matrix $M$ which is invariant under translation, i.e. $M(n, m)=M(n+p, m+p)$ for any integer $p$. Periodic boundary conditions will be assumed, i.e. $n$ and $m$ are defined modulo $N$. It is well known that the elements of the eigenvectors $\left|u_{k}\right\rangle$ have the form

$$
u(n)=\text { Const } \times \exp (i k n)
$$

where $k$ is such that $\exp (i k N)=1$ (see also chapters by Rodríguez-Carvajal and Bourée and by Schober). This is a familiar, but astonishing property: invariance under a group of translations determines the eigenvectors, whatever the numerical values of the matrix elements! The eigenvalues are readily deduced, and do depend on the matrix elements.

This property is a special case of a general property: if a matrix is invariant under the operations $g$ of a group $G$, any element $g$ transforms any eigenvector $|u\rangle$ into an eigenvector $T(g)|u\rangle$, where the matrices $T(g)$ form a 'representation' of $G$, i.e. $T(g) T\left(g^{\prime}\right)=T\left(g g^{\prime}\right)$. In the previous example, the elements of $G$ are the translations $g_{p}$ which transform $M(n, m)$ into $M(n+p, m+p)$. The matrices $T(g)$ are $1 \times 1$ matrices, i.e. numbers, $T\left(g_{p}\right)=\exp (i k p)$. The corresponding representation is said to have dimension 1 . There are as many representations as values of $k$, i.e. $N$. They can be labelled by an index, $T_{k}\left(g_{p}\right)=\exp (i k p)$.

With 2 representations $T$ et $T^{\prime}$ one can build a representation which associates to $g$ the matrix

$$
\left[\begin{array}{cc}
T(g) & 0 \\
0 & T^{\prime}(g)
\end{array}\right]
$$

Such a representation, where all off-diagonal blocks vanish, is said reducible.

When looking for the eigenvectors of a matrix which is invariant under a group $G$, one can impose that they transform as a particular irreducible representation $i$. They are said to 'belong' to representation $i$. Of course all irreducible representations $i$ must be considered, but this method yields all eigenvectors. This property is the expansion theorem [11].

The advantage of the method is that, while the matrix $M$ may be huge, the vectors which belong to each irreducible representation are often given by the diagonalisation of a quite small matrix.

Group theory provides recipes to obtain this small matrix. Such a recipe is outlined in Appendix B. The justification and examples will be found in the chapters by Canals and Schober, by Ballou, and by Rodríguez-Carvajal and Bourée.

Exercise. Consider the wave functions of an electron in a periodic potential. According to the Floquet-Bloch theorem, they have the form $\psi_{k}(r)=\exp (i \mathbf{k . r}) u_{k}(\mathbf{r})$ where $u_{k}(\mathbf{r})$ has the period of the potential. Relate this property to the irreducible representations of the group of lattice translations.

\section{EXAMPLES OF APPLICATIONS OF GROUP THEORY}

\subsection{Degeneracy of a Hamiltonian with a discrete spectrum or matrix}

We shall address the case of a $2 \times 2$ matrix (e.g. the Hamiltonian of a spin 1/2) whose elements are $a_{i j}$. It is degenerate if, and only if it is a multiple of the unit matrix. The conditions for this are $a_{11}=a_{22}$ and $a_{12}=a_{21}=0$. Even if the matrix is Hermitian (as a Hamiltonian should be) these conditions are difficult to fulfill and require the variation of more than a single parameter.... unless both eigenvectors belong to different representations. Then, $a_{12}$ et $a_{21}$ always vanish and degeneracy takes place when $a_{11}=a_{22}$. 


\section{Contribution of Symmetries in Condensed Matter}


Figure 2. a) If 2 eigenvalues of a matrix belong to different representations, the variation of a single parameter $u$ can make them equal. b) If both eigenvalues belong to the same representation, they are generally never equal.

The case of a larger matrix is more complicated, but the conclusion is the same. The variation of a single parameter generally does not lead to degeneracy except for eigenvalues which belong to different representations.

\subsection{Jahn-Teller effect}

Suppose that the two above mentioned eigenvalues are the possible energies of a localized electron in a crystal, whose symmetry is high enough, so that both eigenvalues are degenerate. (As said above, this implies that the 2 eigenvectors belong to 2 different representations). If the crystal turns out to be distorted, there is no longer any degeneracy. Thus one eigenvalue becomes lower and the other one becomes higher. The electron chooses the lower one, so that a distorsion is energetically favourable. This property is studied in detail by Amara [3] and in Refs. [4, 5] together with other properties of the crystal field.

\subsection{Phonon spectrum in a crystal (see chapter by Schober)}

The frequencies of phonons of wave vector $\mathbf{q}$ are the eigenvalues of a finite matrix whose elements are generally not accurately known. However, the symmetry of the matrix is known. If the vector $\mathbf{q}$ is in general position, the matrix has no symmetry and group theory is useless, but if $\mathbf{q}$ is on a symmetry axis or a symmetry plane, it is very useful, for instance it allows to know if there is some degeneracy.

\subsection{Magnetic structures}

When the atoms of a crystal bear a magnetic moment, these moments generally order at low temperature. The resulting magnetic structure superposes to the crystallographic structure. Two cases are possible. Examples of both cases are reviewed in the chapter by Rodríguez-Carvajal and Bourée.

First case: the magnetic structure and the crystallographic structure have a common periodicity (with a unit cell which may be larger than the crystallographic one in the high temperature, paramagnetic region). The symmetry operations which conserve both the magnetic structure and the crystallographic structure form a group. The resulting magnetic groups can be classified in a similar way as the non magnetic space groups (see chapter by Rodríguez-Carvajal and Bourée). Their number is large, but finite.

Second case: the magnetic structure has a period which is incommensurable with the crystal lattice. The most favourable situation is just below a continuous transition from paramagnetism to the magnetically ordered phase. According to Landau's theory of phase transitions (see chapter by 
Toledano) the magnetic structure should correspond to an eigenvector of the generalized, paramagnetic susceptibility matrix, which defines the linear response of the moments to a space-dependent field. In view of the lattice periodicity, the eigenvectors correspond to a sinusoidal field and a sinusoidal magnetic structure, with a wave vector $\mathbf{k}$. The largest eigenvalue of the susceptibility matrix, which diverges at the transition, corresponds to a particular eigenvector of $\chi(\mathbf{k})$. This vector defines the actual period of the magnetic structure. The corresponding eigenvector of the susceptibility matrix defines the nature of the magnetic order. It should belong to a particular irreducible representation of the paramagnetic space group. The use of representation theory is explained in the works of Bertaut [6] complemented by those of Schweizer [7, 8] (see also chapter by Schweizer). At lower temperature, various phenomena may complicate the description. First, harmonics appear as a result of magnetocrystalline anisotropy. Second, lock-in takes place. This means that the magnetic structure becomes commensurable with the crystal lattice, with a period which can be extremely long.

Incommensurate phases are not particular to magnetism. Other examples are mentioned in the chapters by Toledano and by Perez-Mato et al.. Lock-in phenomena can give rise to a 'staircase' of transitions when a parameter (temperature, pressure) is varied. The staircase can be 'devilish' [9] or 'harmless' [10].

\subsection{Superfluid helium 3 and non-conventional superconductivity (see chapter by Houzet)}

Superconductivity is characterized by a non vanishing value of $\Delta_{\sigma \sigma^{\prime}}(\mathbf{k})=\left\langle c_{k \sigma} c_{-k \sigma^{\prime}}\right\rangle$ where the operator $c_{k \sigma}$ annihilates an electron of momentum $\hbar \mathbf{k}$ and spin component $\sigma$ in a particular direction.

In the original theory of superconductivity of Bardeen, Cooper and Schrieffer, the Cooper pairs are in the singlet spin state and the order parameter $\Delta_{\sigma \sigma^{\prime}}(\mathbf{k})$ is independent of the direction $\mathbf{k} / k$. It is practically 0 away from the Fermi surface, and has a value $\Delta$ for $k=k_{F}$. This is a good description of many usual superconductors, e.g. $\mathrm{Nb}$.

In more complicated, 'non-conventional' superconductors, $\Delta_{\sigma \sigma^{\prime}}(\mathbf{k})$ may depend on $\hat{k}=\mathbf{k} / k$ (see chapter by Houzet). Furthermore, Cooper pairs may be in the triplet spin state. When the operations of the point group $\mathcal{G}_{0}$ of the crystal act upon $\mathbf{k}$, the function $\Delta_{\sigma \sigma^{\prime}}(\mathbf{k})$ should transform as an irreducible representation of $\mathcal{G}_{0}$. Thus, group theory provides a classification of possible types of superconductivity. This classification is of great help for experimental research.

In superfluid ${ }^{3} \mathrm{He}$, a similar pairing between fermions of momenta $\mathbf{k}$ and -k occurs. Pairs are in the triplet spin state. As seen later in subsection 7.3, the orbital wave function should transform as an irreducible representation $Y_{\ell m}(\theta \varphi)$ of the rotation group, where $\theta$ and $\varphi$ are the Euler angles of $\mathbf{k}$. The usual antisymmetry condition on the parity of the two-fermion wave function imposes that $\ell$ be odd. In practice, $\ell=1$.

The above enumeration will be complemented and explained in the next chapters of this book. The reader can also find additional information in textbooks, e.g. Refs [11-13].

\section{WAVE FUNCTIONS AND ROTATION GROUP}

\subsection{Eigenfunctions of a Hamiltonian invariant under rotation}

The three-dimensional space is invariant under translation and rotation. The group of translations is too simple for group theory to be necessary. What kind of information can be obtained from invariance under rotation? The problem may be, for instance, to compute the wave function of an electron subject to the attraction of a nucleus situated at a point $\mathrm{O}$, e.g. a hydrogen atom. This is a well-known problem, but it may be of interest to reexamine it at the light of group thory. As seen in section 5, the search for the wave functions (solutions of Schrödinger's equation) can appropriately make use of the irreducible representations of the rotation group. This is actually done in textbooks of quantum mechanics, but often without mentioning it. 


\section{Contribution of Symmetries in Condensed Matter}

Explicitly or not, textbooks state that irreducible representations of odd dimension $(2 \ell+1)$ of the rotation group can be obtained by using as basis vectors (in the terminology of section 5) the functions

$$
Y_{l m}(\theta, \varphi)=B_{l m} P_{l m}(\cos \theta) \exp (\operatorname{im} \varphi)
$$

where the numbers $B_{l m}$ are normalisation constants and

$$
P_{l m}(w)=\left(1-w^{2}\right)^{|m| / 2} d^{|m|} P_{l}(w) / d w^{|m|}
$$

where $P_{l}(w)$ is the Legendre polynomial of order $l$

$$
P_{l}(w)=\left(2^{-l} / l !\right) d^{l}\left(w^{2}-1\right)^{l} / d w^{l}
$$

Group theory says that the solutions of Schrödinger's equation in a rotation invariant potential have the form

$$
\psi_{l m}(r, \theta, \varphi)=R_{l}(r) Y_{l m}(\theta, \varphi)
$$

where the only remaining task is the determination of the radial function $R_{l}(r)$. The factorisation (7.4) is in textbooks, of course, but it is often presented as a miracle while it can be deduced from standard group theory.

\subsection{Rotation and angular momentum}

The spherical harmonics $Y_{l m}(\theta, \varphi)$ are often introduced in textbooks [14, 15] as instruments to study angular momentum, and the relation with rotations is not always mentioned. The end of this section will be devoted to this relation. One has to ask how a function $f(x, y, z)$ (a wave function or anything else) transforms under a rotation of the axes, e.g. around the $z$ axis which is therefore invariant. If $\varphi$ is the rotation angle, the new coordinates are $x^{\prime}=x \cos \varphi+y \sin \varphi$ and $y^{\prime}=-x \sin \varphi+y \cos \varphi$. The function $f(x, y, z)$ transforms into a function of $x^{\prime}, y^{\prime}$ and $z$ which, for a small value of $\varphi$, may be written as

$$
f\left(x^{\prime} \cos \varphi-y^{\prime} \sin \varphi, x^{\prime} \sin \varphi+y^{\prime} \cos \varphi, z\right)=f\left(x^{\prime}-y^{\prime} \varphi, x^{\prime} \varphi+y^{\prime}, z\right)=\left(1+\rho_{z}\right) f\left(x^{\prime}, y^{\prime}, z\right)
$$

where the infinitesimal rotation operator $\rho_{z}$ is $\rho_{z}=x \partial y-y \partial x$. This is also $i L_{z} / \hbar$, where $\mathbf{L}$ is quantum mechanical expression of the orbital angular momentum. Infinitesimal rotation operator $\rho_{x}$ and $\rho_{y}$ around the axes $x$ and $y$ can be derived similarly. The identity $\rho_{\alpha}=i L_{\alpha} / \hbar$ holds for the 3 components $\alpha=x, y, z$. The commutation rules follow, namely

$$
\left[\rho_{x}, \rho_{y}\right]=-\rho_{z} ;\left[\rho_{y}, \rho_{z}\right]=-\rho_{x} ;\left[\rho_{z}, \rho_{x}\right]=-\rho_{y}
$$

The relation between angular momentum and rotation operators will be seen to be a particular case of Noether's theorem addressed in Section 11.

In this section, several concepts and results (spherical harmonics, commutation rules), usually introduced through quantum mechanics, have been derived from group theory and geometry. The geometrical approach is actually simpler and more elementary, since De Broglie's relation $\mathbf{p}=i \hbar \nabla$ (a bold physical hypothesis whose inventor got the Nobel prize) is not needed. It is also more general and more elegant.

\subsection{Eigenfunctions of a Hamiltonian invariant under rotation and translation}

The case treated in subsection 7.1 was that of a localized system. A somewhat different problem is that of a pair of particles linked together by a rotation-invariant attractive potential, but free to move in space. This problem appears for instance in the theory of superfluid ${ }^{3} \mathrm{He}$. 
The wave function in that case is a function $\psi\left(\mathbf{r}_{1}-\mathbf{r}_{2}\right)$ which depends only on the difference between the positions $\mathbf{r}_{1}$ and $\mathbf{r}_{2}$ of the two particles. Since it does not depend on $\left(\mathbf{r}_{1}+\mathbf{r}_{2}\right)$, and is therefore invariant under translation, it is of interest to introduce the Fourier transform (where $V$ is the volume containing the system)

$$
\begin{aligned}
\Phi\left(\mathbf{k}_{1}, \mathbf{k}_{2}\right) & =V^{-1} \int d^{3} r_{1} \int d^{3} r_{2} \exp \left[-i\left(\mathbf{k}_{1} \cdot \mathbf{r}_{1}\right)+i\left(\mathbf{k}_{2} \cdot \mathbf{r}_{2}\right)\right] \psi\left(\mathbf{r}_{1}-\mathbf{r}_{2}\right) \\
& =V^{-1} \int d^{3} r_{2} \exp \left[-i\left(\mathbf{k}_{1}+\mathbf{k}_{2}\right) \cdot \mathbf{r}_{2}\right] \int d^{3} r_{1} \exp \left[i \mathbf{k}_{1} \cdot\left(\mathbf{r}_{1}-\mathbf{r}_{2}\right)\right] \psi\left(\mathbf{r}_{1}-\mathbf{r}_{2}\right) \\
& =\delta_{\mathbf{k}_{1},-\mathbf{k}_{2}} \Phi(\mathbf{k})
\end{aligned}
$$

where

$$
\Phi(\mathbf{k})=\int d^{3} r \exp \left[-i \mathbf{k}_{1} \cdot \mathbf{r}\right] \psi(\mathbf{r})
$$

If the interaction between the two particles is invariant under rotation, the eigenfunctions $\psi\left(\mathbf{r}_{1}-\mathbf{r}_{2}\right)$ of the Hamiltonian have the form (7.4), i.e. $\psi(\mathbf{r})=f(r) Y_{\ell m}(\theta, \varphi)$, where $\theta$ and $\varphi$ are the Euler angles of r. As will now be seen, this implies that the Fourier transform (7.7) has the form

$$
\Phi(\mathbf{k})=F(k) Y_{\ell m}(\theta, \varphi)
$$

where $\theta$ and $\varphi$ are now the Euler angles of $\mathbf{k}$. Indeed, if $\mathbf{k}$ and $\mathbf{r}$ are transformed by the same rotation, the product k.r and the exponential $\exp (i \mathbf{k} . \mathbf{r})$ are conserved. Therefore, if a particular linear relation is satisfied by a function $F(\mathbf{r})$ together with the function $\mathcal{R} F(\mathbf{r})$ obtained from $F(\mathbf{r})$ by applying a rotation $\mathcal{R}$, then the same relation is satisfied by the Fourier transforms. For instance, if $F(\mathbf{r})$ is an eigenfunction of $L_{z}$, its Fourier transform is an eigenfunction of $L_{z}$ for the same eigenvalue. If a set of functions of $\mathbf{r}$ form an irreducible representation of SO3, their Fourier transforms also form an irreducible representation of SO3, actually the same representation. The property (7.8) follows from these two properties. Indeed, if a set of physically acceptable (i.e. finite, continuous and twice differentiable everywhere) functions of $\theta$ and $\varphi$ form an irreducible representation of SO3, it consists of spherical harmonics $Y_{\ell m}(\theta, \varphi)$. This is proved in textbooks $[14,15]$.

Note that, for instance, $L_{z}=i \hbar\left(k_{x} \partial / \partial k_{y}-k_{y} \partial / \partial k_{x}\right)$ when acting on a function of $\mathbf{k}$, while $L_{z}=$ $-i \hbar(x \partial / \partial y-y \partial / \partial x)$ when acting on a function of $\mathbf{r}$.

The commutation rules (7.5) are essential features of rotation operators. They should be the starting point of the search for representations of SO3. This search naturally leads to the concept of spinor as will now be seen.

\section{IRREDUCIBLE, SPINOR REPRESENTATIONS OF EVEN DIMENSION OF THE ROTATION GROUP AND SPIN}

Formula (7.1) yields only irreducible representations of odd dimension of the rotation group. The present section is a quest for irreducible representations of even dimension.

Can one find, for instance, an irreducible representation of dimension 2? Such a representation should contain three $2 \times 2$ matrices associated with infinitesimal rotations around the axes $x, y, z$. These matrices should satisfy commutation relations analogous to (7.5). As well known from textbooks of quantum mechanics, such matrices are the Pauli matrices

$$
\sigma_{x}=\left[\begin{array}{cc}
0 & 1 \\
1 & 0
\end{array}\right] ; \quad \sigma_{y}=\left[\begin{array}{cc}
0 & -i \\
i & 0
\end{array}\right] ; \quad \sigma_{z}=\left[\begin{array}{cc}
1 & 0 \\
0 & -1
\end{array}\right]
$$




\section{Contribution of Symmetries in Condensed Matter}

except that they should be multiplied by $i / 2$. Thus, one may hope that the matrices

$$
\left.\exp \left[(i \varphi / 2)\left(\alpha \sigma_{x}+\beta \sigma_{y}+\gamma \sigma_{z}\right)\right]=1 \cos \varphi / 2+i\left(\alpha \sigma_{x}+\beta \sigma_{y}+\gamma \sigma_{z}\right) \sin \varphi / 2\right)
$$

form a representation of the rotation group $\mathrm{SO} 3 .^{3}$

For physicists, they do. The matrix (8.2) is associated with a rotation of angle $\varphi$ around an axis parallel to a unit vector of components $\alpha, \beta, \gamma$.

However, formula (8.2) has a strange property. The rotation of angle $2 n \pi$ around any axis is clearly the identity. And, for $\varphi=2 n \pi$, the matrix (8.2) is equal to 1 if $n$ is even (which is fine) but it is equal to -1 if $n$ is odd. More generally, relation (8.2) associates to any rotation two matrices $A$ and $-A$. This is not allowed by the definition of a representation accepted by mathematicians.

We shall say that the matrices (8.2) form a 'spinor representation' of SO3. A spinor representation is doubly valued, and therefore, stricto sensu not a representation. However, it will sometimes be called so in the following sections for the sake of simplicity.

The spinor representation acts on two-dimensional column matrices which are called spinors. The word 'spinor' is justified because these objects are used for the mathematical description of a spin 1/2. However, the concept of a spinor was invented by the mathematician Élie Cartan in 1913 [16], 9 years before the experimental discovery of the electron spin by Stern and Gerlach in 1922. The Mathematics were there, ready to be used by theoretical physicists, including Pauli and Dirac.

In section 7, we were using group theory to obtain the eigenvectors of a given operator acting on a known vector space. In the present section, group theory works in a different way. The vector space is $a$ priori not known, it is suggested by group theory.

\section{RELATIVISTIC WAVE FUNCTIONS OF AN ELECTRON}

Relativity uses a 4-dimensional space, in which the changes of coordinates combine time $t$ (or rather $\left.x^{0}=c t\right)^{4}$ and the 3 space coordinates $x^{\alpha}(\alpha=1,2,3)$. These changes of coordinates constitute the Poincaré group. Among these transformations, those which conserve the origin form the Lorentz group.

While the definition of a wave function in non-relativistic physics involves representations of the group SO3 of three-dimensional rotations, one expects that in relativistic physics representations of the Lorentz group are needed. Only the case of a single particle of spin 1/2 will be addressed here. Then the appropriate irreducible representation turns out to be of dimension 2 and spinorial, i.e. doubly valued. It involves Pauli matrices and will not be explicitly derived here. Instead, emphasis will be put on a problem which is absent in non-relativistic quantum mechanics.

It turns out that one cannot define a wave function which transforms according to the irreducible spinor representation of dimension 2. One needs [17-19] a four-dimensional wave function (i.e. a column matrix of 4 elements) which transforms according to a reducible spinor representation of dimension 4 , obtained by combining the irreducible representation of dimension 2 with an analogous, but not equivalent representation. This is explained in Appendix C. The wave function satisfies Dirac's equation, which in the absence of electromagnetic field reads

$$
\hbar \gamma^{\mu} \partial_{\mu} \psi+m c \psi=0
$$

where summation over $\mu$ is implied according to Einstein's convention. The $4 \times 4$ matrices $\gamma^{\mu}$ verify anticommutation rules $\left\{\gamma^{\mu}, \gamma^{\nu}\right\}=2 g^{\mu \nu}$, where

$$
g^{\mu \nu}=g^{\mu} \delta^{\mu \nu}
$$

\footnotetext{
3 The equality (8.2) can be checked by expanding the exponential in power series and using the anticommutation rules of the Pauli matrices.

4 The upper indices denote so-called contravariant components of a quadrivector $x$, while lower indices denote covariant components. The relation between both is $x^{\alpha}=g^{\alpha \gamma} x_{\gamma}$, where $g^{\alpha \gamma}$ is defined by (9.1) and summation over $\gamma$ is implied according to Einstein's convention.
} 
$\operatorname{and}^{5}$

$$
g^{1}=g^{2}=g^{3}=1 ; g^{0}=-1
$$

A possible choice is

$$
\gamma^{\alpha}=\left[\begin{array}{cc}
0 & -i \sigma_{\alpha} \\
i \sigma_{\alpha} & 0
\end{array}\right] \quad(\alpha=1,2,3) ; \quad \gamma^{0}=i\left[\begin{array}{cc}
1 & 0 \\
0 & -1
\end{array}\right]
$$

where blocks are $2 \times 2$ matrices and $\sigma_{i}$ denotes Pauli matrices.

\section{ELECTROMAGNETISM AND ELECTRODYNAMICS: GAUGE SYMMETRY}

Electromagnetic radiation is characterised by an electric field and a magnetic field, which are 3dimensional vectors. They can be considered as the components of an antisymmetric tensor $F_{\mu \nu}$ of order 4. It is preferable to express this tensor from the 4 components $A_{\mu}$ of the vector potential.

$$
F_{\mu \nu}=\partial_{\mu} A_{\nu}-\partial_{\nu} A_{\mu}
$$

The use of the vector potential, which has only 4 components, is the best choice. However, it is not unambiguously defined. Indeed the physically meaningful, measurable quantities are the fields, and they are not modified if $A$ is replaced by

$$
A_{\mu}^{\prime}=A_{\mu}-g_{\mu} \partial_{\mu} \Lambda(\mathbf{r}, t)
$$

where $g_{\mu}$ is defined by $(9.2)^{6}$. The arbitrary choice of $\Lambda$ is called a gauge. Quantification of the electromagnetic field (10.1) leads to photons, which are a special case of gauge bosons. This concept turns out to be a basic one in elementary particle physics.

If now a charged particle interacts with the electromagnetic field, this is the domain of electrodynamics. The choice of the gauge is still arbitrary, but now the gauge transformation (10.2) implies a change of the phase of the wave function (see appendix D). These changes form the group $\mathrm{U}(1)$.

It is noteworthy that a similar phase plays an essential role in superconductivity and superfluidity. Indeed the Hamiltonian is left invariant by a global change of the phase of the field operators, and this invariance is broken in the superfluid or superconducting phase. It is often said that in those materials the gauge invariance is broken (see chapter by Tolodano). However, this 'gauge' is physically different from the electromagnetic gauge addressed above. In particular, photons play no part.

Other generalisations of the concept of gauge in condensed matter physics have been addressed in a rather thick book by Kleinert [20]. For instance, it naturally appears in the theory of elasticity if dislocations or other defects are present.

Gauge transformations in electrodynamics form a group. This remark in itself is not very useful since the abelian group U(1) does not justify the use of the heavy machinery of group theory. Indeed, excellent textbooks on quantum electrodynamics do not even mention the concept of gauge group. However, electrodynamics provide a didactic introduction to much more elaborate gauge theories which describe elementary particles, where group theory is essential. Before coming to that, it is appropriate to give 2 general theorems, useful in condensed matter physics as well as in elementary particle physics.

\footnotetext{
5 The quantity $g^{\mu \nu}$ is a tensor, which has the same diagonal form in all frames related by Lorentz transformations. On the contrary $\delta^{\mu \nu}$ is not a tensor and $g^{\mu}$, as defined by (9.2), is not a vector. Relation (9.2) can be true with a particular particular choice of axes, but is not invariant under a Lorentz transformation. For that reason the quantity $g^{\mu}$ is generally not introduced. However it will be useful in section 10 .

${ }^{6}$ As remarked previously, $g^{\mu}$ is not invariant under a Lorentz transformation. Therefore, $\Lambda(\mathbf{r}, t)$ is not necessarily invariant either. The frequently used 'Coulomb gauge' is not.
} 


\section{TWO GENERAL THEOREMS}

\subsection{Noether's theorem (1915)}

To any continuous symmetry group a conserved quantity may be associated and vice-versa [21, 22]. Thus momentum conservation may be regarded a consequence of translation symmetry in space, and angular momentum conservation is related to invariance under rotation.

\subsection{Goldstone's theorem (1961)}

In condensed matter physics, Goldstone's theorem states that if a continuous symmetry is broken, then new excitations should appear with a vanishing 'gap'. For instance, a crystal breaks the continuous translation of space. The corresponding excitations are transverse acoustic phonons, the frequency $\omega_{q}$ of which is related to the wave vector $\mathbf{q}$, for small $q$, by the linear relation $\omega_{q}=\alpha q$. Their energy thus vanishes when $q$ goes to 0 . Goldstone's theorem $[23,24]$ states that this property is general. In the case of magnetism, the symmetry under spin rotation is broken at low temperature, and the consequence is the appearance of magnons. However, these magnons are only gapless if the Hamiltonian is invariant under spin rotation, and this implies that there is no magnetic anisotropy.

The case of superconductivity is addressed in Appendix F.

Although condensed matter physicists frequently invoke Goldstone's theorem, Goldstone was concerned by elementary particles. His proof was relativistic and made use of a technical formalism which was appreciably more elaborate than that needed in condensed matter physics. Now, in relativity, the energy of a particle is $E=c\left(m^{2} c^{2}+p^{2}\right)^{1 / 2}$. If the mass at rest $m$ is finite, this energy writes, if $p$ is small, $E=m c^{2}+p^{2} /(2 m)$; but if the mass is 0 , then $E=c p=c \hbar q$. Therefore, for physicists of elementary particles, the favourite formulation of Goldstone's theorem is that, if a continuous symmetry is broken, then there should be new massless bosons ${ }^{7}$.

Vanishing or non-vanishing mass? This determines the range of the interaction carried by 'gauge bosons'. Let the case of the electromagnetic field be considered first. It is transported by photons, which have 0 mass. A charge at the origin O produces at distance $r$ an electric potential $V(r)$ proportional to $1 / r$, solution (for $r \neq 0$ ) of the Laplace equation $\nabla^{2} V=0$. But if the potential is caused by particles of mass $m \neq 0$, it should verify Schrödinger's equation $\nabla^{2} V=\kappa^{2} V$ with $\kappa=m c / \hbar$ [25]. The solution is $V(r)=$ Const $\times \exp (-\kappa r) / r$, it has a finite range.

As will be seen, Goldstone's theorem was a puzzle for elementary particle physicists between 1960 and 1970 .

\section{ELEMENTARY PARTICLES}

\subsection{Natural questions: parity, etc.}

In condensed matter physics, the number of electrons is assumed to be constant, just as the number of nuclei of each element. The only relevant interaction is assumed to be the electromagnetic interaction. This is a good approximation on the Earth, even though in certain cases the presence of a radioactive substance requires using some nuclear physics. In nuclear physics, nucleons are bound together by another interaction which is called the 'strong' nuclear interaction. The binding energy of a nucleon in a nucleus is about $10 \mathrm{MeV}$, much higher than typical energies in condensed matter physics. For instance, the cohesive energy of a metal is between 1 and $10 \mathrm{eV}$. When studying elementary particles, for instance the interaction between quarks, typical energies are of the order of the GeV. This is the order of

\footnotetext{
${ }^{7}$ In condensed matter physics too, massless quasi-particles as phonons have a linear spectrum, $\omega_{q}=\alpha q$, while particles with a non-vanishing mass, for instance electrons, have a quadratic spectrum.
} 


\section{EPJ Web of Conferences}

magnitude of the strong interaction. But there are other nuclear interactions, called weak, which account for instance for beta radioactivity.

Are these interactions subject to the same invariance rules which are usual in condensed matter physics?

For instance, the equations which we write in condensed matter physics are invariant under the time reversal operation $T$ and by the reversal $P$ of space coordinates. Is that true when particles are able to decay and combine?

The answer is yes for the strong interaction. Moreover, the strong interaction is invariant with respect to another operator $C$, unknown in condensed matter physics. This operator changes a particle into an antiparticle (for instance an electron into a positon). These invariance properties limit possible reactions and simplify the theory of the strong interaction.

However weak interactions are neither invariant under $P$, nor $C$. For some time it was believed to be invariant under the product $C P$ but it is not quite true neither. However the invariance under $C P T$ is ensured by a theorem proved in 1954 by Lüders and Pauli using very safe hypotheses.

\subsection{More surprising concepts: isospin, etc.}

High energy theoreticians have introduced many other symmetries, which they hope to be helpful to restrict the proliferation of particles.

Before going farther, one can ask whether it is useful to talk so much about elementary particles in a school about symmetry in condensed matter.

A first reason to do so is that the interaction between both kinds of physicists has been quite productive. Certain scientists as Wilson, Anderson [26], Parisi, Yang, Lee have been successful in both fields.

A second reason is that, in a school on symmetry held in 2009, it is difficult to ignore the Nobel prize awarded in 2008 to Yoichiro Nambu, Makoto Kobayashi and Toshihide Maskawa for their work on symmetry in elementary particle physics.

The symmetries introduced by elementary particle theorists are not so natural as those generally used in condensed matter physics. A pioneering idea was that of Heisenberg, who suggested in 1932 that there is a symmetry between the proton and the neutron.... provided the electromagnetic interaction, which is weak at the scale of the nucleus, is ignored. Taking only the strong interaction into account, the proton and the neutron can indeed be regarded as two states of a same particle, the nucleon. Similarly to the two states of a ferromagnet, which differ by the spins of the electrons, one defines an analogous variable, called isospin. Like the spin of the electron, it has 2 possibles eigenvalues. One corresponds to the proton, the other one to the neutron. The Lagrangian of the strong interaction, as Heisenberg conceived it, had to be invariant under SU(2), and this turns out to yield 2 eigenvalues for the fundamental representation, as it should.

\subsection{Quarks and gluons}

After Heisenberg, the theory of elementary particles became more complicated. Protons and neutrons lost their dignity of elementary particles and split into 3 quarks. The first theory of quarks was worked out in particular by Gell-Mann in the third quarter of the twentieth century. It is described in some detail in the appendix E. Quarks interact by exchanging gluons, as electrons exchange photons. Like photons, gluons have zero mass. Like photons, they are subject to gauge invariance, but now the gauge group is no longer the abelian group $\mathrm{U}(1)$, it is $\mathrm{SU}(3)$. Similarly to photons which carry the electromagnetic interaction, gluons carry the strong interaction between quarks. From this interaction between quarks results a strong interaction between nucleons, which, with respect to the interaction between quarks, is somehow what the van der Waals interaction between atoms is with respect to the electromagnetic interaction. There are 6 quarks, characterized by 6 "flavours", but 2 flavours (called up et down, $u$ et $d$ ) are sufficient to build a proton of formula $u u d$ or a neutron of formula $u d d$. The two states postulated 


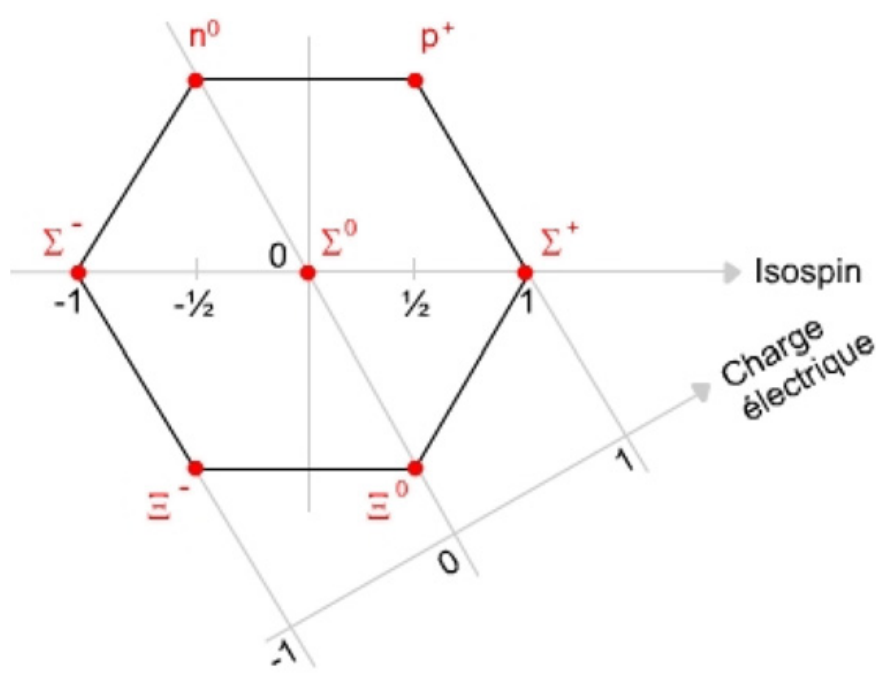

Figure 3. A picture showing the approximate symmetry between particles if only the strong interaction are taken into acccount. The isospin concept, proposed by Heisenberg, introduced a symmetry between the proton and the neutron. The scheme has then be complemented by introducing particles $\Sigma$ and $\Xi$, which are combinations of quarks, as neutrons and protons are.

by Heisenberg result, as well as the invariance under $S U(2)$. Introducing the so-called strange quark, other particles may be obtained, whose symmetry can be represented by figure 3 .

\subsection{The standard model and weak interactions}

After having built theories of the strong interaction, theoreticians of particles included them, together with quantum electrodynamics, into a more unified theory called standard model, which also include weak interactions. The standard model is a gauge theory, which relies on a gauge group $S U(3) \times$ $S U(2) \times U(1)$. As has been seen, $\mathrm{SU}(3)$ corresponds to the strong interaction and $\mathrm{U}(1)$ corresponds to electromagnetism. Does that mean that $\mathrm{SU}(2)$ describes weak interactions? It is more complicated, because the $S U(2) \times U(1)$ symmetry spontaneously breaks. It remains a U(1) symmetry, with massless gauge bosons, which are photons. But the gauge bosons which correspond to the SU(2) symmetry have acquired a mass. These are the bosons of the weak interaction $\left(\mathrm{W}^{+}, \mathrm{W}^{-}\right.$et $\left.\mathrm{Z}^{0}\right)$.

However, new massless bosons might be expected, namely those that Goldstone's theorem associates with a continuous broken symmetry. Experimentally, these bosons are not observed. This paradox has been resolved by the introduction of a new, massive boson, called Higgs boson. It results from a recombination of the Goldstone boson, which in fact does not appear. This 'Higgs mechanism' is a property of gauge symmetries [27-31]. In the solution of the problem, an essential contribution was that of Nambu, who was awarded the Physics Nobel prize in 2008. The Higgs mechanism is described in some detail in Appendix F.

While Goldstone's theorem was devised for elementary particles, it turns out to have no exact application in that field. An approximate application is provided by pions, which have a small mass and indeed are approximate Goldstone bosons, related to the violation of an approximate symmetry with respect to chirality.

\subsection{Beyond the standard model: supersymmetry, etc.}

The standard model is probably correct, since all its predictions have been experimentally checked apart from the Higgs boson which has not yet been observed. However, one would like to have a 


\section{EPJ Web of Conferences}

more predictive model. For instance, the standard model does not explain why the proton charge is equal to that of the electron in absolute value. This question mark is one of the reasons which have motivated grand unification theories, based for instance on $\mathrm{SU}(5)$ or $\mathrm{SO}(10)$. The Higgs mechanism is repeatedly used and multiplies the number of elementary particles, which had been somewhat reduced by interpreting hadrons as bound states of quarks.

Symmetry between bosons and fermions is also popular. This is supersymmetry.

This does not solve the problem of incorporating gravitation : present hopes rely on string theory. This theory goes beyond the concept of point particles, which is rather nice. Unfortunately this implies to explore a space with 10 dimensions.

\subsection{Matter and antimatter}

Since there is an approximate symmetry between matter and antimatter, one might expect to have approximately the same quantity of matter and antimatter in the world. Moreover, the violation of the symmetry under the charge conjugation operator $\mathrm{C}$ is not sufficient to explain the absence of antimatter. As shown by Sakharov in 1967, CP symmetry should also be violated. During years, astronomers have searched the heaven in their quest for an anti-Universe. The quest stopped in 1964 when an experiment on kaons revealed CP symmetry violation. Matter and antimatter are not formed in the same proportion. During the Big Bang, matter and antimatter annihilated, some matter remained, but no antimatter. Then began the quest for the reason of this symmetry violation. In 1972, Makoto Kobayashi and Toshihide Maskawa exploited an idea of Nicola Cabibbo to obtain the answer. Their theory implied increasing the number of quarks, which had already raised to 4 thanks to Glashow, Iliopoulos, \& Maiani [32]. Completing the miracle of multiplication of quarks, Kobayashi and Maskawa added two other quarks (bottom and top) whose existence was experimentally confirmed a few years later.

While the description of interacting electrons and photons by quantum electrodynamics is a well defined theory, the theory of other particles is not yet completed. Various theories have been proposed, and none is perfectly satisfactory, either because they do not explain all what theorists would like to explain, or because they rely upon hazardous hypotheses. A common point to all theories is the extensive use of symmetry.

\section{CONCLUSION}

Symmetry plays an essential role in physics. Sometimes it is an intuitive property. For instance, a mere glance at a crystal may give an idea of its symmetry. In other instances it is a result of the deep reflexions of scientists. Gauge invariance and gauge groups are examples.

Symmetry breaking is also an essential concept. It is a natural idea in the field of phase transitions. Much less in theories of elementary particles, where it is partly the result of the imagination of theoreticians in their (honourable) quest for unification.

In this introduction, other important issues have been left aside. An example is fluid mechanics, where symmetry breaking is frequently observed. Examples are the Rayleigh-Plateau instability (fragmentation of a cylindric flow resulting from surface tension) or the Rayleigh-Bénard instability (formation of convection rolls when a fluid is heated from below).

Groups generated by transformations are not the only groups present in physics. Permutation groups are other examples. Bose and Fermi statistics associate to permutations of $N$ identical particles two different irreducible representations, but they are so simple that group theory is not required. However, group theory can be useful if one looks for other statistics, which are neither Bose nor Fermi. They have been discovered, but only in two dimensions. Particles which are neither bosons nor fermions have been called anyons $[33,34]$.

A few words should be said about the 'renormalization group', which is a very efficient method in theoretical physics. Its elements are transformations acting on functions. As a matter of fact all the transformations have not an inverse, so that the so-called 'renormalization group' is rather a semi-group. 


\section{Contribution of Symmetries in Condensed Matter}

After these digressions to high energy physics, anyons and renormalisation group, the time has come to return to condensed matter, which is the topic of this school. Symmetry is a natural companion in this field, and it can facilitate the solution of many problems. The next speakers will present the recipes that group theory provides, these recipes are efficient, but the student will perhaps require to work hard before he or she is able to use them.

But group theory is not only a tool to solve problems. It is also a beautiful theory which improves the insight on many concepts of theoretical Physics.

The author is grateful to Jean Iliopoulos and Pierre Fayet for two elementary lectures on elementary particles, to Roger Balian, Manuel Houzet and Micha Zhitomirskii for helpful explanations and to Vincent Michal for a careful reading of the final version of this review.

\section{APPENDIX A. ELECTRONIC STRUCTURE IN A HEXAGONAL LATTICE. DIRAC POINTS IN GRAPHENE}

Graphene is a single graphite monolayer. Its band structure has a remarkable peculiarity which will be shown in this appendix. In the so-called tight binding approximation, the Hamiltonian of a single electron is

$$
\mathcal{H}=\sum_{i j} \gamma_{i j} c_{i}^{+} c_{j}
$$

where $i$ and $j$ denote the sites of the graphene honeycomb lattice, $c_{i}$ and $c_{i}^{+}$respectively destroy and create an electron at site $i$, and the numbers $\gamma_{i j}$, which describe the possibility to jump from a site to another, have the symmetry properties of the lattice. The energies of the electronic states are the eigenvalues of the matrix $\Gamma$ formed by the $\gamma_{i j}$. If there were a single site per unit cell, the eigenvalues and eigenvectors would be obtained by a Fourier transformation. However, the honeycomb lattice has two atoms per cell. It is made of two planar, entangled triangular Bravais lattices 1 and 2. The components $u^{i}$ of an eigenvector can be written as $u_{i}=u_{1} \exp (i \mathbf{k} . \mathbf{R})$ if $i$ is point $\mathbf{R}$ of lattice 1 , and $u_{i}=u_{2} \exp (i \mathbf{k} . \mathbf{R})$ if $i$ is point $\mathbf{R}$ of lattice 2 . Thus $u_{R \alpha}=u_{\alpha} \exp (i \mathbf{k} . \mathbf{R})$ with $i=1$ or 2 . The components $u_{1}$ and $u_{2}$ are eigenvectors of the matrix

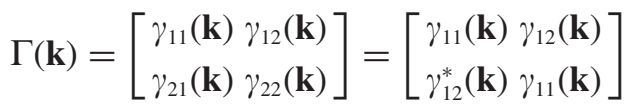

where

$$
\gamma_{\alpha \gamma}(\mathbf{k})=\sum_{R^{\prime}, \gamma} \exp \left[i \mathbf{k} \cdot\left(\mathbf{r}_{R \alpha}-\mathbf{r}_{R^{\prime} \gamma}\right)\right]
$$

In a first step the lattice 2 will be ignored. At point $\mathbf{R}$ of lattice 1 (Fig. 4a), the wave function has the complex value $u_{1} \exp (i \mathbf{k} . \mathbf{R})$. Instead, one can consider the two-dimensional real vector $u_{1} \mathbf{v}(\mathbf{R})$, where

$$
\mathbf{v}(\mathbf{R})=[\cos (\mathbf{k} \cdot \mathbf{R}), \sin (\mathbf{k} . \mathbf{R})]
$$

It is natural to look for a vector field $\mathbf{v}(\mathbf{R})$ which has the same rotation symmetry of order 3 as the lattice itself. Such a field does exist, as seen from Figure $4 \mathrm{~b}$. If $\mathrm{O}$ is the center of any of the triangles, the structure (b) is invariant by a rotation of $2 \pi / 3$ around $O$, together with a rotation of the vecteurs $\mathbf{v}$ of $2 \pi / 3$ too. A consequence of this invariance is

$$
\sum_{R} \gamma_{O R} \mathbf{v}(\mathbf{R})=0
$$




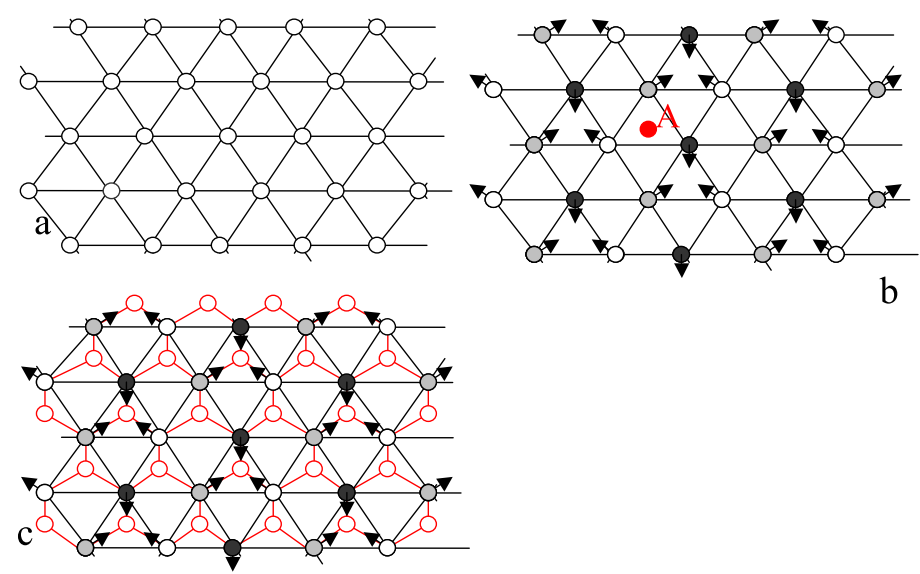

Figure 4. a) A planar, triangular lattice. b) A vector field which preserves the 3-fold rotation symmetry. c) Superposing another triangular lattice, a honeycomb lattice is obtained. In every site of the second lattice, the 'molecular field' (A.4) produced by the first lattice vanishes.

where $\mathbf{R}$ designates the sites of lattice 1. The structure of Figure $4 \mathrm{~b}$ is consistent with (A.3) provided $\mathbf{k}$ is equal to $\mathbf{K}$ given by

$$
K_{x}=\frac{2 \pi}{3 b} ; \quad K_{y}=\frac{2 \pi}{b \sqrt{3}},
$$

where $b$ is the distance between the sites of the triangular lattices. As a function of the atomic distance $a=b / \sqrt{3}$ on the honeycomb lattice,

$$
K_{x}=\frac{2 \pi}{3 a \sqrt{3}} ; \quad K_{y}=\frac{2 \pi}{3 a},
$$

Relation (A.4) can therefore be written as

$$
\gamma_{12}(\mathbf{K})=0
$$

Thus both eigenvalues of the matrix $\Gamma(\mathbf{K})$ are equal, whatever the range of the $\gamma_{i j}$. The electronic states $\epsilon^{-}(\mathbf{k})$ and $\epsilon^{+}(\mathbf{k})$ have a common value $\epsilon(\mathbf{K})$ for $\mathbf{k}=\mathbf{K}$ Actually, with a very good approximation, $\gamma_{i j}$ may be assumed different from 0 only for neighbouring sites $\mathrm{i}$ and $\mathrm{j}$ (so that one site is on lattice 1 and the other on lattice 2). In that case, if $\mathbf{k}$ is $\mathbf{K}$, the electronic states verify $\epsilon^{-}(\mathbf{k})<\epsilon(\mathbf{K})<\epsilon^{+}(\mathbf{k})>\epsilon(\mathbf{K})$. Since, in pure graphene, there is exactly one electron per site, there is a full band and an empty band which touch in a discrete number of points of the reciprocal space. These points are called Dirac points.

\section{APPENDIX B. RECIPE TO OBTAIN THE EIGENVALUES OF A MATRIX WHICH BELONG TO A PARTICULAR REPRESENTATION}

This recipe will only be given in the case of a representation $i$ of dimension 1. This representation associates to each element $g$ of the group a number $\alpha_{i}(g)$ which should be of absolute value 1 . Otherwise the modulus of $\alpha_{i}\left(g^{s}\right)=\alpha_{i}^{s}(g)$ would go to $\pm \infty$ when the exponent $s$ goes to $\infty$. Thus, $\alpha_{i}(g)=\exp \left[i \varphi_{i}(g)\right]$. A theorem of group theory states that

$$
\sum_{g} \exp \left\{i\left[\varphi_{i}(g)-\varphi_{j}(g)\right]\right\}=n \delta_{i j}
$$

where $n$ is the number of elements $g$, and all representations are assumed of dimension 1 . 


\section{Contribution of Symmetries in Condensed Matter}

The matrix $M$ acts on a $N$-dimensional vector space $\mathcal{V}$. In this vector space the choice of basis vectors is arbitrary. We want to choose basis vectors $e_{i \alpha}$ such that each of them belong to the irreducible representation $i$, i.e. $g$ transforms $e_{i \alpha}$ into $e_{i \alpha} \exp \left[i \varphi_{i}(g)\right]$. According to group theory, this is possible. Thus, each vector $v$ of $\mathcal{V}$ may be written as

$$
v=\sum_{i \alpha} v_{i \alpha} e_{i \alpha}
$$

The trick is now to multiply by $g$ and by $\exp \left[-i \varphi_{i}(g)\right]$, and sum over $g$. One obtains

$$
\sum_{g} g v \exp \left[-i \varphi_{i}(g)\right]=\sum_{j \alpha} v_{j \alpha} \sum_{g} g e_{j \alpha} \exp \left[-i \varphi_{i}(g)\right]=\sum_{j \alpha} v_{j \alpha} e_{j \alpha} \sum_{g} g \exp \left\{i\left[\varphi_{j}(g)-\varphi_{i}(g)\right]\right\}
$$

or, using (B.1),

$$
\sum_{g} g v \exp \left[-i \varphi_{i}(g)\right]=n \sum_{\alpha} v_{i \alpha} e_{i \alpha}
$$

Thus one obtains a linear combination of the basis vectors $e_{i \alpha}$ which belong to representation $i$. Other combinations can be obtained by taking $N$ independent vectors $v$. The method yields all vectors $e_{i \alpha}$ belonging to representation $i$. Each of them is generally obtained several times, but this has little importance. The important point is that, if there are $N_{i}$ independent vectors (or column matrices) $e_{i \alpha}$, the eigenvectors of $M$ have the form

$$
u_{i \alpha}=\sum_{\gamma} x_{\alpha \gamma} e_{i \alpha}
$$

and the coefficients $x_{\alpha \gamma}$ are the eigenvectors of a $N_{i} \times N_{i}$ matrix instead of a much bigger, $N \times N$ matrix.

\section{APPENDIX C. SPINOR REPRESENTATIONS OF THE LORENTZ GROUP, DIRAC SPINORS, AND DIRAC'S EQUATION}

Two-dimensional, spinor representation of the Lorentz group.

To obtain it, it is appropriate to associate to any 4-vector $\mathbf{x}=(c t, x, y, z)$ the two-dimensional, Hermitian matrix

$$
X=\left[\begin{array}{cc}
c t+z & x+i y \\
x-i y & c t-z
\end{array}\right]=t+x \sigma_{x}+y \sigma_{y}+z \sigma_{z}
$$

It is readily seen that $\operatorname{det} X=c^{2} t^{2}-x^{2}-y^{2}-z^{2}$ is the Minkowskian norm of $\mathbf{x}$.

It will then be shown that the $2 \times 2$ complex matrices $B$ of determinant 1 constitute a representation of the Lorentz group. To do that, we consider the matrix.

$$
X^{\prime}=B X B^{+}
$$

where $B^{+}$denotes the Hermitian conjugate of $B$. The matrix $X^{\prime}$ is Hermitian and its determinant is equal to $\operatorname{det} X$. Therefore it defines a 4 -vector $\mathbf{x}^{\prime}$ through formula (C.1). Therefore (C.2) is a Lorentz transformation.

For instance a translation in the $z$ direction corresponds to $B=\exp \left(-\varphi \sigma_{z} / 2\right)$. Indeed, using the anticommutation properties of the Pauli matrices, it it seen that

$$
\begin{aligned}
B X B^{+} & =B X B=\exp \left(-\varphi \sigma_{z} / 2\right)\left(c t+x \sigma_{x}+y \sigma_{y}+z \sigma_{z}\right) \exp \left(-\varphi \sigma_{z} / 2\right) \\
& =\left(c t+z \sigma_{z}\right) \exp \left(-\varphi \sigma_{z}\right) \exp \left(-\varphi \sigma_{z} / 2\right)+\left(x \sigma_{x}+y \sigma_{y}\right) \exp \left(-\varphi \sigma_{z} / 2\right)
\end{aligned}
$$


This is a Lorentz transformation which corresponds to a uniform translation along the $z$ axis, with velocity $v=\beta c$ and $\sinh \varphi=1 / \sqrt{1-\beta^{2}}$.

A similar calculation shows that a rotation of angle $\varphi$ around the $z$ axis corresponds to $B=\exp \left(-i \varphi \sigma_{z} / 2\right)$ as in the non relativistic case.

Similar rules apply to rotations around $x$ and $y$ and to translations along $x$ and $y$. This demonstrates that any Lorentz transformation corresponds to a matrix B. Actually two matrices since $B$ can be replaced by $-B$ in (C.2).

In the above defined representation of the Lorentz group, the operator $\sigma_{\alpha}$ describes both an infinitesimal rotation around the $\alpha$ axis and a slow translation along this axis. The only difference is the $i$ factor. Since both operations are physically completely different, the two-dimensional spinor representation is not appropriate for a relativistic quantum description.

Dirac representation of the Lorentz group.

If the matrices $B(g)$ form a representation of a non commutative group $G$ whose elements are $g$, the Hermitian conjugate matrices $B^{+}(g)$ generally do not form a representation, because $B^{+}(g) B^{+}\left(g^{\prime}\right)=B^{+}\left(g^{\prime} g\right)$ is generally different from $B^{+}\left(g g^{\prime}\right)$. However, the matrices $\left[B^{+}(g)\right]^{-1}$ do form a representation. If $G$ is the Lorentz group and $B(g)$ are the matrices defined above, the two representations are not equivalent. Indeed, the equivalence of two representations $B$ and $B^{\prime}$ implies $B^{\prime}(g)=U B(g) U^{-1}$ and therefore $\operatorname{Tr} B=\operatorname{Tr} B^{\prime}$. This relation is not fulfilled, for instance for a diagonal matrix, $B_{11}^{\prime}+B_{22}^{\prime}=\left(B_{11}^{*}+B_{22}^{*}\right) /\left(B_{11} B_{22}\right)^{*}=B_{11}^{*}+B_{22}^{*}$ is generally not equal to $B_{11}+B_{22}$.

Dirac's representation associates to any Lorentz transformation $g$ the $4 \times 4$ matrix

$$
\left[\begin{array}{cc}
B(g) & 0 \\
0 & {\left[B^{+}(g)\right]^{-1}}
\end{array}\right]
$$

This reducible representation acts on a 4-dimensional spinor space, and the wave function which obeys Dirac's equation is an element of this space. It is formed by 2 two-dimensional spinors, one of which transforms as $B$, the other as $\left(B^{+}\right)^{-1}$

\section{APPENDIX D. GAUGE INVARIANCE IN ELECTRODYNAMICS}

Tne case of a single electron interacting with the electromagnetic field will be considered. The electron (of mass $m$ and charge $q=-e$ ) is represented by a four-component wave function (the spinor of Dirac's equation) and the electromagnetic field by the 4-component vector potential vecteur $A_{\mu}$. In their textbook, Jauch \& Rohrlich [35] write the following function which they call 'Lagrangian' (which, however, is called Lagrangian density by other authors):

$$
\mathcal{L}=-(1 / 2) \partial_{\mu} A_{\nu} \partial^{\mu} A^{\nu}-\bar{\Psi}\left(\gamma_{\mu} \partial^{\mu}+m\right) \Psi-i e \bar{\Psi} \gamma_{\mu} A^{\mu} \Psi
$$

where $\gamma_{\mu}$ denotes the four $4 \times 4$ matrices defined in Section 9, and summation is to be done on indices (Einstein's convention).

The Lagrangian (A.1) allows to define the action $S=\int \mathcal{L} d^{4} x$, where the integral is over the domain between 2 hyperplanes of the relativistic space-time. Testa [18] notices that the Lagrangian (D.1) is invariant under the transformation (10.2) combined with the transformation of the electronic wave function $\Psi(\mathbf{x})$ into

$$
\Psi^{\prime}(\mathbf{x})=\exp [i \Lambda(\mathbf{x})] \Psi(\mathbf{x})
$$

Dirac's equation cannot easily be generalized to more than one electron. It is therefore of interest to derive (D.2) from Schrödinger's equation, This is done by Cohen-Tannoudji et al. [36], pages 169 and 170. 


\section{APPENDIX E. A FORMALISM FOR THE STRONG INTERACTION}

Quarks, having spin 1/2 as electrons, are also represented by a four-component spinor $\psi$. However, they also have a 'flavour' and a 'colour' which require an index $p=1,2, . ., 6$ for the flavour and an index $i=1,2,3$ for the colour. They interact via gauge bosons called gluons, which are massless as photons. They are represented by a tensor field labelled by $\lambda, \mu=0,1,2,3$, and an additional index $a$. The Lagrangian has a form which looks like that of the electromagnetic field, except that there are more indices because the gauge group is more complicated.

$$
\mathcal{L}_{Q C D}=\bar{\Psi}_{i p}\left(i \gamma^{\mu} \partial_{\mu}-m_{p}\right) \Psi_{i p}-g G_{\mu}^{a} \bar{\Psi}_{i p} \gamma^{\mu} T_{i j}^{a} \Psi_{j p}-(1 / 4) G_{\mu \nu}^{a} G_{a}^{\mu \nu}
$$

The 'Gell-Mann matrices' $T_{i j}^{a}$ are given by group theory. The coupling constant $g$ is a parameter. With the 6 masses $m_{p}$, the theory has 7 parameters. Since the 6 masses are different, symmetry might be expected to be absent from the problem. However, the masses of the quarks $u$ and $d$ are of the order of the $\mathrm{MeV}$ (apart from a factor $c^{2}$ ), which is weak with respect to the masses of the nucleons which are of the order of the $\mathrm{GeV}$. Thus there is a good approximate symmetry between proton and neutron (if electromagnetism is ignored). The situation becomes worse when the other quarks are taken into account, since they are appreciably heavier (a few $100 \mathrm{MeV}$ to a few $100 \mathrm{GeV})$.

\section{APPENDIX F. LONG RANGE INTERACTIONS AND THE HIGGS MECHANISM}

In a neutral gas, acoustic waves have a linear spectrum, i.e. a frequency $\omega_{q}=c q$. What happens if the particles are electrically charged? Sound waves become plasmons, and those plasmons now have a non vanishing frequency when the wave vector number $q$ vanishes. Let the proof be briefly recalled. The equation of motion is $d^{2} u_{q} / d t^{2}=-V_{q} q^{2} u_{q}$ where $u_{q}$ is the Fourier transform of the displacement and $V_{q}$ is the Fourier transform of the potential. For short range interactions, $V_{q}$ is finite for $q=0$, and the frequency $\omega_{q}$ is proportional to $q$. For Coulomb interactions, $V_{q}$ is proportional to $1 / q^{2}$ and the plasma frequency does not vanish for $q=0$.

This remark was the starting point of an article published by Anderson in 1963. The author also addressed the analogous case of superconductivity. The Goldstone would then be an oscillation of the current, $j(x, t)=j_{0} \cos \left(q x-\omega_{q} t\right)$. But such an oscillation would produce a space charge, with a non-vanishing energy at vanishing wave number $q$ because of the long ranged Coulomb interaction. The Goldstone mode of superconductivity has a non-vanishing frequency for $q=0$. It is therefore not a real Goldstone mode. In the language of elementary particles, it is 'massive'.

Anderson was able to see the analogy between the finite plasma frequency, the lacking Goldstone mode in a superconductor, and the absence of the expected Goldstone boson in the Zoo of elementary particles.

\section{References}

[1] P. Curie, Bulletin de la Société minéralogique de France 7 (1884) 418 and Oeuvres de Pierre Curie, available through Gallica (http://gallica.bnf.fr/) p. 79

[2] B. Wunsch, F. Guinea, F. Sols, New Journal of Physics 10 (2008) 103027.

[3] slides of the lecture M. Amara gave at the school (http://www.ill.eu/news-events/pastevents/2009/ecole-theorie-des-groupes/transparents-cours-td-tp/, file "19-AMARA-Crystal Field Effect On Atomic States.pdf"').

[4] H. Bethe, Annalen der Physik 32 (1929), 133-208; English translation in Selected works of Hans A. Bethe: With Commentary, Hans A. Bethe, in World Scientific Series in 20th century Physics, Vol. 18, 1996. 
[5] M. Tinkham, Group Theory and Quantum Mechanics, McGraw-Hill, New York, 1964.

[6] E.F. Bertaut, Journal de Physique Colloque C1 32 (1971) 462.

[7] J. Schweizer, C.R. Physique 6, 375 and Corrigendum (2006) C.R. Physique 7 (2005) 823.

[8] J. Schweizer, J. Villain, A.B. Harris Eur. Phys. J. Appl. Phys. 38 (2007) 41.

[9] F. Axel, S. Aubry, J. Phys. C: Solid State Phys. 14 (1981) 5433.

[10] J. Villain, M. Gordon, J. Phys. C: Solid State Phys. 13 (1980) 3117.

[11] H.W. Streitwolf (1971) Group theory in solid-state physics (Macdonalds, London).

[12] P.H.E. Meijer, E. Bauer (2004) Group Theory: The Application To Quantum Mechanics (Dover).

[13] M. Hamermesh (1962) Group theory and its application to physical problems (Dover).

[14] L.I. Schiff (1955) Quantum Mechanics (McGraw-Hill, New York).

[15] C. Cohen-Tannoudji, B. Diu, F. Laloë (1997) Mécanique quantique (Hermann, Paris).

[16] E. Cartan, Bull. Soc. Math. France 41 (1913) 56.

[17] E. Cartan (1938) Leçons sur la théorie des spineurs. Paris, H. Hermann.

[18] M. Testa (1993) Articles 'gauge, teorie di' and 'spinore' in Enciclopedia delle Scienze fisiche, tome 2, p. 842 (Rome).

[19] A. Comtet (2005) L'équation de Dirac Lecture at the Université Pierre et Marie Curie. http://cel.archives-ouvertes.fr/cel-00092970/fr/

[20] H. Kleinert (1989) Gauge fields in condensed matter World scientific, Singapore.

[21] E. Noether, "Invariante Variationsprobleme", Nachr. d. König. Gesellsch. d. Wiss. zu Göttingen, Math-phys. Klasse (1918), 235-257; English translation M. A. Travel, Transport Theory and Statistical Physics 1(3) 1971,183-207.

[22] N. Byers (1996) http://fr.arxiv.org/abs/physics/9807044.

[23] J. Goldstone, Nuovo Cimento 19 (1961) 154.

[24] J. Goldstone, A. Salam, S. Weinberg, Phys. Rev. 127 (1962) 965.

[25] H. Yukawa (1949) Nobel lecture. http://nobelprize.org/

[26] P.W. Anderson, Phys. Rev. 130 (1963) 439.

[27] P.W. Higgs, Phys. Lett. 12 (1964) 132.

[28] P.W. Higgs, Phys. Rev. Lett. 13 (1964) 508.

[29] P. Higgs, C. R. Physique 8 (2007) 970.

[30] F. Englert, R. Brout, Phys. Rev. Lett. 13 (1964) 321.

[31] R. Brout, F. Englert, C. R. Physique 8 (2007) 973.

[32] S. L. Glashow, J. Iliopoulos, L. Maiani, Phys. Rev. D 2 (1970) 1285.

[33] F. Wilczek, Phys. Rev. Lett. 49 (1982) 957.

[34] Monroe, Don, New Scientist, 1 October 2008.

[35] J.M. Jauch, F. Rohrlich (1955) The theory of photons and electrons (Addison-Wesley, Cambridge, U.S.A.).

[36] C. Cohen-Tannoudji, J. Dupont-Roc, G. Grynberg (1987) Photons et atomes (EDP Sciences, CNRS editions, Les Ulis). 